\title{
Mechanical scanning in intravascular ultrasound imaging: artifacts and driving mechanisms
}

\author{
H. ten Hoff ${ }^{a}{ }^{1}$, E.J. Gussenhoven ${ }^{b}$, A. Korbijn ${ }^{a}$, F. Mastik ${ }^{a}$, C.T. Lancée ${ }^{a}$, N. Bom ${ }^{b}$ \\ ${ }^{a}$ Erasmus University Rotterdam, NL-3000 DR Rotterdam, The Netherlands \\ bInteruniversity Cardiology Institute of The Netherlands
}

Received 30 June 1994; revision received 20 January 1995; accepted 23 January 1995

\begin{abstract}
Objective: Currently, intravascular ultrasound (US) imaging catheters are developed and produced to provide a complementary diagnostic method in the treatment of blood vessel obstructive disease. Typical catheter dimensions are a diameter of $1-2.5 \mathrm{~mm}$ and a length of $1-1.5 \mathrm{~m}$. A real-time $360^{\circ}$ US scan of the surrounding of a catheter tip can be obtained by mechanical rotation of a US beam, approximately perpendicular to the catheter axis and originating from a single element. It is the purpose of this paper to make the reader aware of the various image artifacts that might occur with flex-shaft mechanical scanning and of the limitations of the technique, and to discuss the possible causes as well as alternative drive mechanisms. Methods: The study is based on definition and discussion of various error mechanisms caused by improper transmission of rotation flex-shaft drives. Adequate ultrasonic imaging implies a oneto-one relationship between the catheter tip scan rotation angle and the angle of polar image line deflection on the display. This can be achieved by applying a predictable, uniform scan rotation at the catheter tip. Deviation from an intentionally uniform scan rotation gives rise to image artifacts, causing either loss of absolute orientation (due to a constant error angle), loss of quality of the real-time aspect (due to a stochastic error angle), or the occurrence of deceptive image distortions (due to a periodic error angle). The magnitude of an acceptable constant tip rotation error (actual tip rotation angle minus expected angle) depends on the need for maintaining an absolute orientation of the image. The stochastic error should be kept to a minimum: random misplacement of image lines in the order of degrees already is disturbing to the interpretation of the image. As a practical arbitrary criterion for the periodic error angle, a maximum peak-to-peak value of $20^{\circ}$ can be defined. Results: Catheter tip driving mechanisms have to be evaluated, considering their potential to maintain a predictable (often uniform) catheter tip scan rotation. A $0.8 \mathrm{~mm}$ OD double-layer spiral type flexible drive-shaft has been developed, which meets the requirements, exhibiting a bending rigidity of 1.5 $\mathrm{Nmm}^{2}$ and a torsional rigidity of more than $100 \mathrm{Nmm}^{2}$. This drive-shaft shows a sensitivity to influences, inducing
\end{abstract}

\footnotetext{
*Corresponding author, Thoraxcentre Ee 2302, Erasmus University Rotterdam, P.O. Box 1738, NL-3000 DR Rotterdam, The Netherlands. Tel.: +31 104088030 ; Fax: +31 104365191 .

${ }^{1}$ Currently employed by Boston Scientific Corp., P.O.Box 7407, Watertown, MA 02272, USA.
} 
rotation error angles, like pre-curvature of the shaft and friction, of about $1 \%$ compared to a simple wire of e.g. stainless steel. Conclusion: For practical purposes, today's technologies in the production of flex-shafts have improved and these devices can be very usefully applied in clinical situations. Flex-shaft problems can be avoided if, alternatively, rotation power could be provided in the catheter tip itself. To this effect, an electromagnetic micromotor has been developed, with a diameter of $1 \mathrm{~mm}$, exhibiting adequate running behaviour. Implementation of this motor in a catheter tip is in progress.

Keywords: Intravascular ultrasound; Image artifact; Flexible drive-shaft; Micromotor

\section{Introduction}

A $360^{\circ}$ cross-sectional echo scan of the surrounding of a catheter tip, showing an image plane approximately perpendicular to the catheter-axis, with a penetration depth of about $10 \mathrm{~mm}$, can be obtained by electronic or mechanical steering of a high frequency $(30 \mathrm{MHz})$ ultrasonic beam and adequate processing of the echo signals (Bom et al. 1989). Advantages and disadvantages of both scanning systems are described in Bom and Roelandt 1992 and Bom et al. 1992. If the system characteristic of image quality is taken as the decisive criterion, the choice in favour of mechanical scanning can be made based on potentially better image resolution and sensitivity. Therefore, in our laboratory the concept of mechanical scanning has been pursued, resulting in the development of a complete intravascular ultrasound imaging system (Fig. 1).

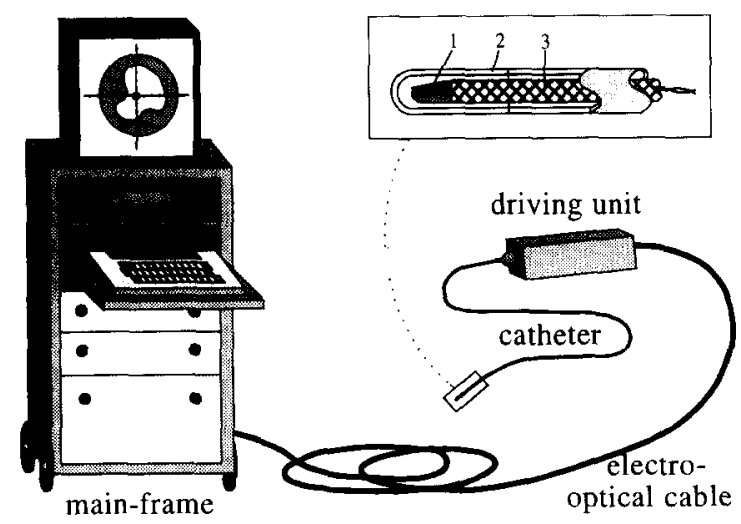

Fig. 1. Intravascular ultrasound imaging system, based on mechanical rotational scanning. US transducer (1), sonolucent dome (2), flexible drive-shaft (3).

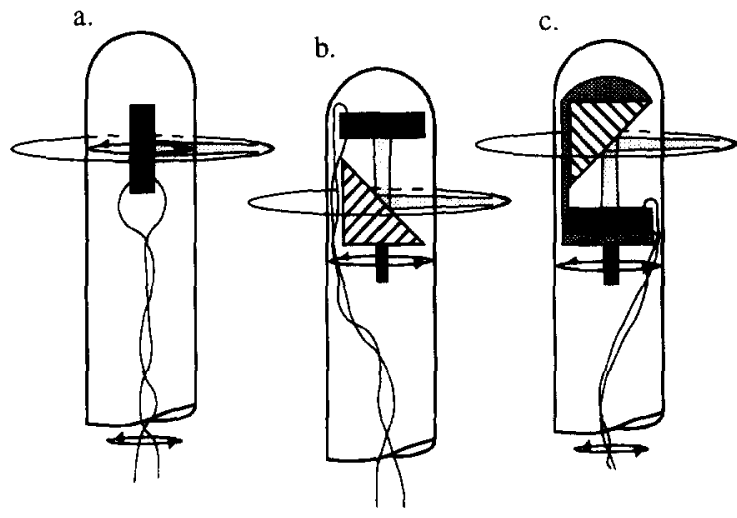

Fig. 2. Catheter tip configurations for mechanical US scanning: (a) Rotating transducer and leads. (b) Rotating deflecting mirror. (c) Rotating transducer, deflecting mirror and electrical leads. In (a) and (c) the conducting leads are designed to be positioned inside the flexible drive-shaft.

A cross-sectional echo scan of the surrounding of a catheter tip is made by mechanically rotating an ultrasonic beam, generated by a single US element. This might be achieved either by rotating a radially emitting element (Fig. 2a) or a deflecting mirror, in which case the US transducer emits axially and is mounted statically in the catheter tip (Fig. 2b) or rotates together with the mirror (Fig. $2 c)$. Transducer rotation requires the rotation of its electrical leads, since slip-rings of these small catheter tip dimensions, transmitting a pulse peak current of several amperes, are unrealistic.

Mechanically rotating parts should be shielded from the patient's arterial wall, hence, a sonolucent enclosing dome is required, consequently calling for the presence of a coupling fluid in the dome to transmit the US energy to and from the transducer. The principal problem to be solved for the mechanically scanning system is the development of an adequate rotation mechanism to drive the 
element or mirror in the catheter tip. Mechanical scanning may give rise to catheter tip motion relative to the vessel wall, and particularly an unpredictable scan motion will introduce image artifacts, influencing the image quality and reliability. It is believed that by good system design these image artifacts can be limited to an acceptable, low level.

Various other aspects of artifact formation have been described in literature (de Kroon et al. 1991; Finet et al. 1993).

\section{Clinical application of intravascular ultrasound}

The necessity for more detailed morphological information about the arterial anatomy and understanding of the ultimate effect of vascular intervention has accelerated the current advances in intravascular ultrasound systems. In vitro studies have shown that this imaging technique provides accurate information on lumen size, vascular anatomy and location of the atherosclerotic lesion. Echographic distinction between elastic and muscular arteries was feasible, based on the composition of the arterial media. Clinical use of intravascular ultrasound showed a characteristic threelayered appearance of the coronary, iliac, femoral and popliteal artery. From the in vitro information, we conclude that the hypoechoic vascular wall corresponds with the media of a muscular artery. The characteristic hypoechoic media facilitates visualization of a superimposed lesion

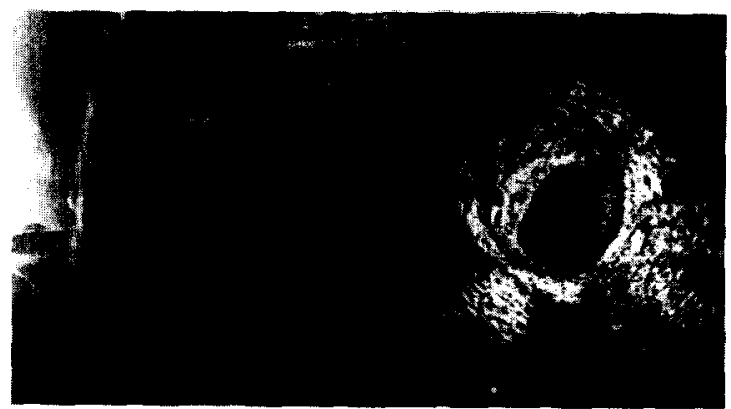

Fig. 3. Angiogram and intravascular ultrasound cross-section of the superficial femoral artery. While angiography at level 11 appeared normal, a significant lesion is seen echographically. The black arrow indicates the media; the white arrow indicates the level of the cross-section at $11 \mathrm{~cm}$

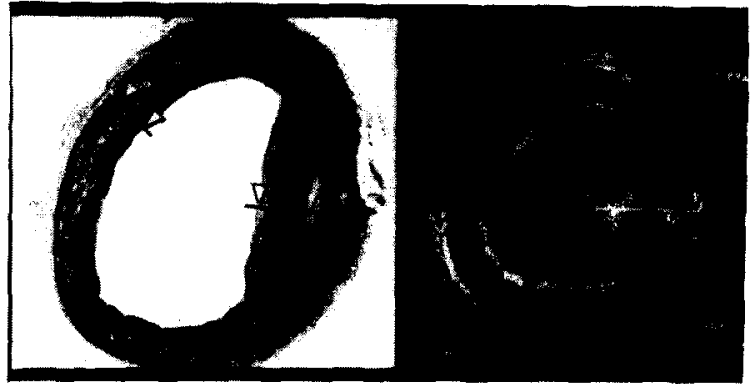

Fig. 4. Histologic and corresponding intravascular ultrasound cross-section showing the influence of lesion on media thickness. At 11 o'clock a media is well seen in the absence of a lesion; at 3 o'clock, the media becomes absent in the presence of a lesion (catheter calibration marks at $1 \mathrm{~mm}$ interval).

(Fig. 3). It was found that intravascular ultrasound accurately determines that medial thickness of muscular arteries is inversely related to lesion thickness (Fig. 4).

Normal vascular morphology could easily be distinguished from cross-sections with pathology, using the hypoechoic media as reference. During pull-back on the ultrasound catheter in the clinical setting, it was observed that the anatomy and extent of the lesion could vary significantly. Lesions were evidenced in cross-sections that were angiographically normal. Angiographically suc-

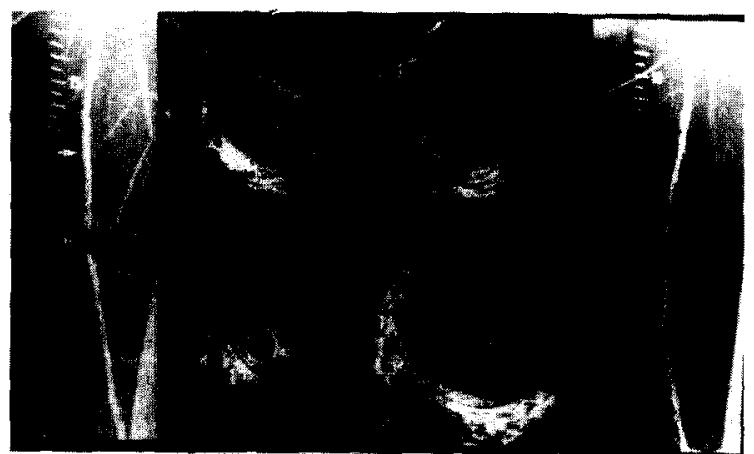

Fig. 5. Angiographic images and intravascular ultrasound cross-sections of the superficial femoral artery obtained before (on the left) and after (on the right) balloon angioplasty. Level 18: not subjected to the intervention; the ultrasound images are unchanged showing $57 \%$ area reduction. Level 22 : ultrasound confirmed a $>90 \%$ obstruction. Following intervention the residual obstruction was $57 \%$. A dissection and vessel rupture were the additional anatomic features seen. 
cessful balloon angioplasty could be accompanied by dissection, plaque rupture and vessel wall rupture, as evidenced by intravascular ultrasound (Fig. 5).

The imaging apparatus used was manufactured by Du-MED (Rotterdam).

\section{Mechanically-induced image artifacts}

The echoes of a transmitted US pulse, received from a certain direction of the acoustic beam axis, are displayed in grey-scale value and represent one radial image line on the display screen. The image line is defined by the echo signals of the pulse, its location of origin and the direction in the crosssectional plane. In general the latter two are not being monitored and, instead, the imaging process is based on a few assumptions. The location of origin of the US pulse coincides with the position of the acoustic element in the catheter tip. This location is assumed not to change within one $360^{\circ}$ scan, with respect to the imaged structures, due to either catheter tip or vessel wall motion. Furthermore, uniform catheter tip scan rotation, with a well-known rev./min, will ensure correct angular correlation of the image lines on the display and a reference direction (e.g. the direction at time $t=0$ ) ensures correlation of the absolute orientation of images. However, these assumptions are not generally valid. The effects of catheter tip motion on the image will be studied in the following.

\subsection{Scan speed and frame rate with respect to vessel wall motion}

Basic specifications of the imaging system are the choices of scan speed and frame rate.

Arteries in vivo pulsate with a frequency of about $1 \mathrm{~Hz}$, transmitting the pressure pulse-wave of the heart. This results in periodic vessel deformations. Some arteries perform (periodic) translations, due to the motion of the structures or organs in which they are imbedded or to which they are attached, and by doing so, change the position of the catheter tip in the vessel. The coronary arteries, for instance, move vigorously with the heart muscle (again with a periodicity of about 1 $\mathrm{Hz}$ ). Both motion mechanisms will cause image artifacts, if the scan speed is too low.

If the amplitude of the relative motion between catheter tip and vessel wall is small enough, image artifacts will be acceptably small, when a scan is produced in less than $1 / 10$ of a motion period.

Dynamic analysis of images is only possible if the frame rate is sufficiently high. The most obvious way to meet both requirements is the application of fast continuous scanning, resulting in a frame rate of more than 10 frames/s. Real-time imaging, showing natural vessel wall motion and deformations smoothly to the eye, can be performed at 25 frames/s. Practical system parameters may limit frame rate.

\subsection{Image artifacts due to radial and tangential catheter tip motion}

Radial and tangential catheter tip motion with respect to the blood vessel originate either from motion of the blood vessel or from the catheter tip itself, in which case the motion is related to the driving motion. The first is already dealt with and is no real problem if the scan speed is high enough.

In practice, the catheter is supported on many points by the tortuous blood vessel, through which it has been advanced. This immobilizes the catheter. The free length of the catheter tip, where it is hardly supported by the vessel wall, is a factor in the possible occurrence of radial tip motion. The catheter tip may also be stabilized by the guide-wire, which runs through its free lumen or to which it is attached by a 'side-saddle' construction.

Naturally, the elimination of the causes of catheter tip motion, which would degrade image quality, is preferred. Therefore, it is important to apply small well-balanced rotating masses, realize well-aligned supports of rotating elements and avoid a rotating frequency close to the fundamental frequency of the bending mode motion of the free tip length. A schematic of image distortion due to periodic radial catheter tip motion, with the same frequency as the driving frequency is shown in Fig. 6.

Tangential catheter tip motion occurs when the catheter tip describes circles around the supposed static location of the catheter in the blood vessel. If the catheter causes this motion, it will be related to the scan rotation in the catheter tip. The effect is an unreal representation of the radial measures of the blood vessel: the lumen will be shown either too small or too large. 
a.

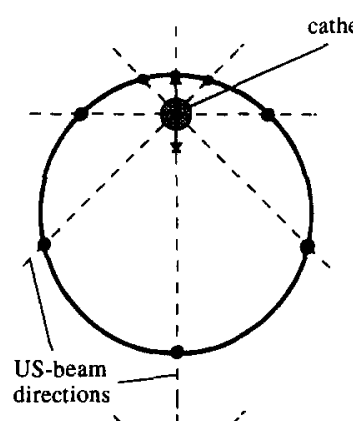

b.

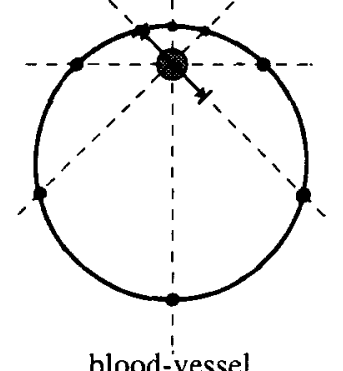

blood-vessel

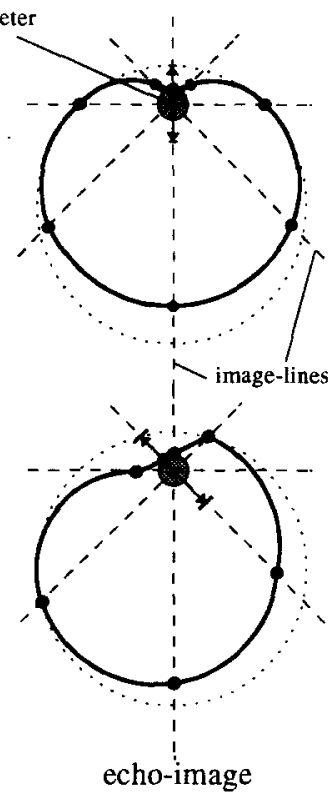

echo-image

Fig. 6. Schematic of image distortion caused by periodic radial catheter tip motion (arrows), in case the periodicity of this motion corresponds with the scan rotation. Radial catheter tip motion perpendicular to the vessel wall (a) and not perpendicular to the wall (b).

\subsection{Image artifacts due to non-uniform catheter tip scan rotation}

In general, the polar image on the display is built up with the assumption of a uniform catheter tip scan rotation. It is difficult to guarantee uniform angular velocity at the catheter tip, although its average angular velocity, determined over a sufficient number of rotations, is kept constant. A rotation error angle, $\theta$ (rad) can be defined as:

$\theta=\psi_{\text {act }}-\psi_{\text {exp }}$

where $\psi_{\text {act }}(\mathrm{rad})=$ the actual tip rotation angle, traversed since time $t=0$ and $\psi_{\exp }(\mathrm{rad})=$ $\omega t=$ the expected rotation angle, assuming uniform catheter tip scan rotation with a given angular velocity $\omega(\mathrm{rad} / \mathrm{s})$.

The unpredictable rotation error angle $\theta$ causes misplacement of image lines on the display, because an image line, recorded at a rotation angle $\psi_{\text {act }}$, is displayed at an angle $\psi_{\text {exp. }}$. This causes image artifacts of several kinds, depending on the

nature of the course of the error angle in time (ten Hoff et al. 1989).

The error angle can have a constant $\left(\theta_{\mathrm{c}}\right)$, a periodic $\left(\theta_{\mathrm{p}}\right)$ and a stochastic $\left(\theta_{\mathrm{s}}\right)$ component. The first causes a rotation of the whole displayed image over an angle $\theta_{c}$, so that without the help of an anatomic 'land-mark' the absolute orientation of the image with respect to the patient's anatomy will be lost. For most present clinical applications this may not be of great importance. The stochastic error causes a mismatch of sequential images, resulting in unstable real-time imaging. This error should be kept very small. Depending on the driving mechanism, the periodic error angle may repeat itself with the driving frequency $\omega$. If other frequency components than the driving frequency or its higher harmonics are present, the effect on the image is similar to that of the stochastic error.

The periodic error angle, which repeats itself every revolution with high precision, may be represented by the periodic function $\theta_{\mathrm{p}}(\omega)$. Sequential images will match, resulting in a sharp real-time image delineation. However, image lines do not appear on screen in accordance with the real cross-sectional geometry of the blood vessel. The effect of the periodic error angle on the image reliability can be studied by simulation, using a sine function to describe the course of the periodic error angle in time.

According to the mechanism causing the error, the periodic rotation error angle $\theta_{\mathrm{p}}(\omega)$ can be represented by a sine function of either the theoretical rotation angle $\psi_{\text {exp }}$, so that:

$\theta_{\mathrm{p}}=\theta_{0} \sin \psi_{\exp }=\theta_{0} \sin \omega t$

or of the actual rotation angle $\psi_{\text {act }}$, so that:

$$
\begin{aligned}
\theta_{\mathrm{p}} & =\theta_{0} \sin \psi_{\mathrm{act}}=\theta_{0} \sin \left(\psi_{\exp }+\theta_{\mathrm{p}}\right) \\
& =\theta_{0} \sin \left(\omega t+\theta_{\mathrm{p}}\right)
\end{aligned}
$$

This renders a recursive definition of the angle error function.

Computer simulations of these effects for various values of the peak-to-peak periodic error angle $\left(=2 \theta_{0}\right)$ are illustrated in Fig. 7 , showing the misplacement in the image of eight equally spaced 


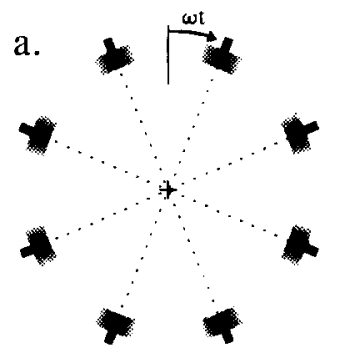

peak-to-peak error angle $0^{\circ}$

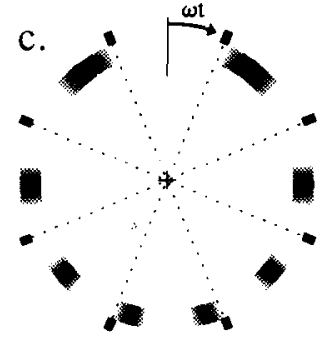

peak-to-peak error angle $50^{\circ}$

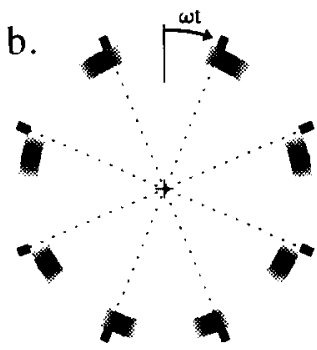

peak-to-peak error angle $20^{\circ}$

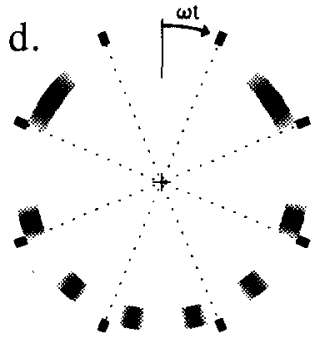

peak-to-peak error angle $80^{\circ}$

Fig. 7. Simulation of the image distortion in case of a sineshaped rotation error angle, as a function of the actual rotation angle $\psi_{\text {act }}$. The ideal image of eight US reflectors equally distributed around the catheter tip, peak-to-peak error angle is $0^{\circ}(\mathrm{a})$. Distorted images of the same reflector configuration when peak-to-peak error angle is $20^{\circ}$ (b), $50^{\circ}$ (c) and $80^{\circ}$ (d). Note regions of expansion and compression of the image.

point reflectors around the catheter tip. Expansion and compression of sectors of the image can be recognized.

The periodic error angle represents a danger to the correct clinical interpretation of the image. Over- or underestimation of the blood vessel occlusion may occur, resulting in the choice of incorrect therapeutic measures. The degree of misinterpretation is, except from the peak-to-peak value of the periodic error angle, also dependent on the blood vessel geometry and the position of the scanning catheter tip in it. A concentric stenosis imaged by a catheter tip positioned in the center, is not sensitive to this problem, but in an asymmetrical situation, illustrated in Fig. 8, quantification of the rate of stenosis will be estimated erroneously. Fig. 8 shows a slightly distorted and a severely distorted US image of an iliac artery, recorded in vitro, where the catheter tip rotation is established by a poorly performing flexible driveshaft transmission.

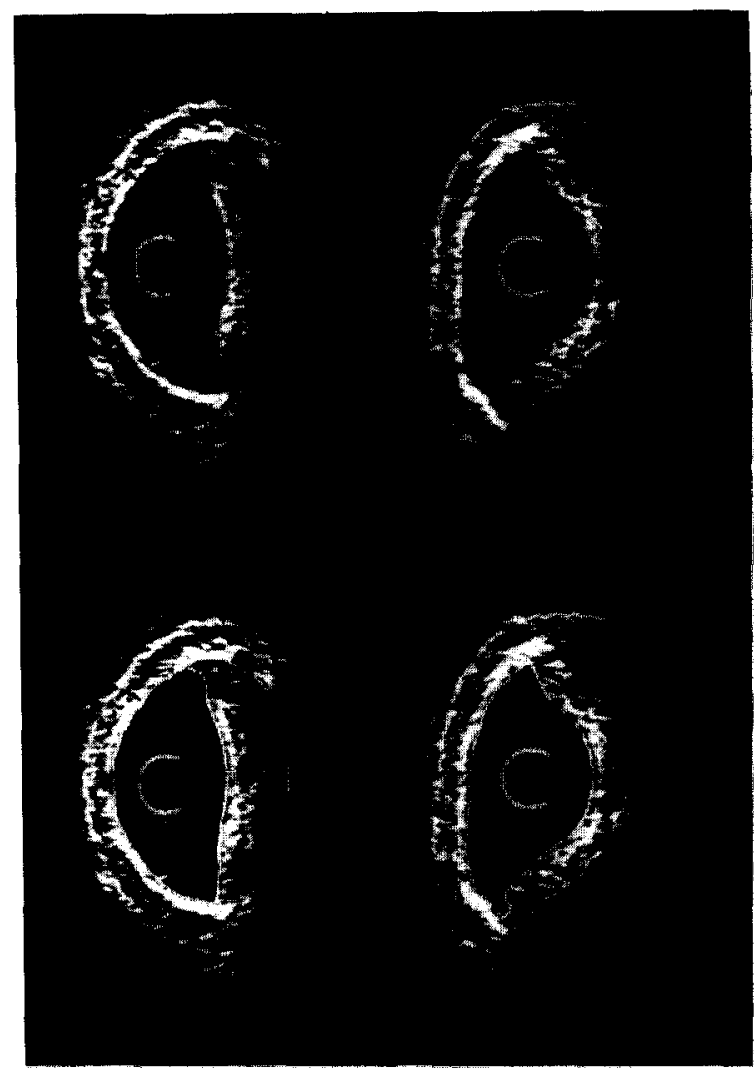

Fig. 8. Distortion of the US image of an iliac artery, in vitro, with apparent obstructive disease. The slightly distorted image of the vessel cross-section (left) suffers from a small periodic scan rotation error of $9^{\circ}$ peak-to-peak. The rate of stenosis is $41 \%$ of the original lumen area of $26.1 \mathrm{~mm}^{2}$. The more distorted image of the same vessel cross-section (right) is recorded at a periodic scan rotation error angle of about $55^{\circ}$ peak-to-peak. It shows a rate of stenosis of $55 \%$ of a supposedly original lumen area of $32.1 \mathrm{~mm}^{2}$. These figures have been determined with contour tracing software (bottom) ( $\mathrm{Li}$ et al. 1991). Note: these images do not represent the imaging performance of flex-shaft driven catheters in general, functioning in the present clinical setting. Here the effects of non-uniform catheter tip scan rotation have been enhanced for illustration purposes.

Another extreme example of non-uniform element rotation leading to artifacts may occur when the catheter tip is forcibly pushed through a pathway which is too narrow. The artifact illustrated in Fig. 9 was caused by an introducer with a too narrow sheath diameter. Images obtained under these circumstances can not be used for quantitative analysis. 


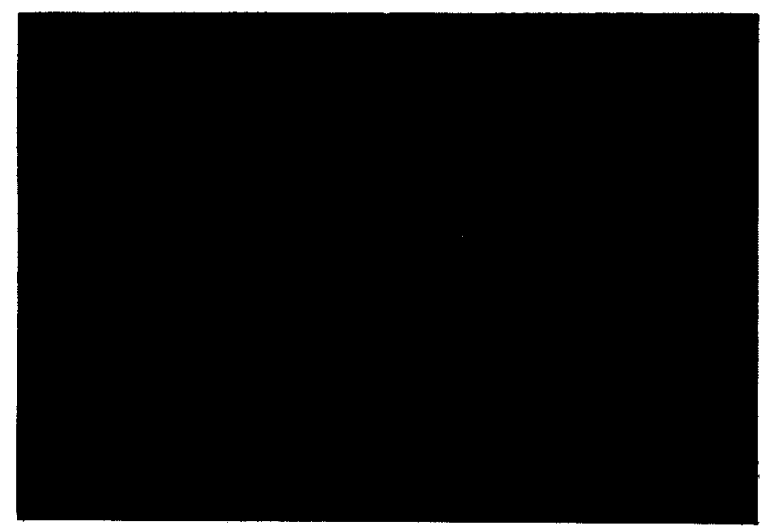

Fig. 9. Example of an artifact due to severe non-uniform element rotation as a result of forces to be avoided on the catheter tip.

\subsection{Specifications for adequate catheter tip scan ro- tation}

A lower limit to the scan speed of $1000 \mathrm{rev} . \mathrm{min}$ is given by the need for real-time imaging and to avoid image artifacts due to relative motion of the imaged objects. Very high scan speeds may cause other problems, as will subsequently be described, so that a maximum limit must also be defined.

Fire-hose effect. High constant or momentary rotation speeds may cause problems due to mismatch of US pulse emission and echo reception beam directions. The sensitivity for echoes from more remote structures will diminish, resulting in a smaller penetration depth of imaging. Furthermore, the image lines (lines of highest echo amplitude) will become slightly curved, causing another image deformation if not corrected for (ten Hoff et al. 1989). This phenomenon is known as the fire-hose effect. Analysis for a penetration depth of $10 \mathrm{~mm}$ (US frequency $30 \mathrm{MHz}$ ) shows that the momentary rotation speed of the US beam should not exceed $10000 \mathrm{rev} . / \mathrm{min}$ in order to avoid this effect.

Data acquisition. The signal processing, designed and built for the echo catheter imaging system, is based on the generation of 500 image lines per revolution and a maximum of 3600 frames $/ \mathrm{min}$. Data acquisition therefore has a maximum speed of 30000 image lines per second. At nominal rotation speeds $>3600 \mathrm{rev} . \mathrm{min}$, maintaining real- time display, the tangential resolution has to be reduced. If these high speeds only occur momentarily and the nominal rotation speed is $\leq 3600$ $\mathrm{rev} . / \mathrm{min}$, the data acquisition should be redesigned or buffers for temporary data storage should be provided.

Constant error angle $\theta_{c}$. As explained earlier, the three components of the scan rotation error angle are not equally important for the image reliability. The constant error, causing the whole image to rotate, may not be that significant, as long as the loss of the absolute orientation of the image with respect to the patient's anatomy is not important.

Stochastic error angle $\theta_{s}$. The stochastic error angle disturbs the real-time aspect of the image and should be minimized by ensuring a high degree of reproducibility of succeeding images. Imaging is performed with 500 radial image lines per revolution, which, in addition to the beam pattern, determines the tangential resolution in the visualization of biological tissue. A misfit of two succeeding images in the order of one degree can therefore already disturb the real-time aspect of the image. The variation $\Delta \bar{\omega}$ of the average angular velocity over one revolution $\bar{\omega}$ should therefore be limited according to:

$$
\frac{\Delta \bar{\omega}}{\bar{\omega}}<\frac{ \pm 0.5}{360}= \pm 0.0014
$$

Velocity variations are caused by small stochastic changes $\Delta T$ in the load $T$, i.e. the total driving torque. The stiffness $S$ of the driving mechanism determines the sensitivity of the momentary angular velocity $\omega$ to the torque variations $\Delta T$. In the worst case situation, where $\Delta T$ exists over the whole time-span of one revolution, this sensitivity is expressed by:

$\Delta \bar{\omega}=\frac{\Delta \mathrm{T}}{\mathrm{S}}$

Analysis of a driving mechanism, functioning at a chosen angular velocity, with a certain driving torque and stiffness and an estimation of occurring disturbances of this torque will show whether the specification of eq. 4 can be met.

Periodic error angle $\theta_{p}$. Periodic errors with a 
frequency one order of magnitude lower than the scan rotation frequency cause the entire image to rotate forwards and backwards over a certain angle. Sensitivity to slightly changing catheter geometry (with the heart beat) can cause this phenomenon. It can be annoying and its effect should be minimized. Periodic errors with higher frequencies, other than the driving frequency or its higher harmonics, should be considered as affecting the image in a way similar to the stochastic errors.

Without knowledge concerning the clinical protocol utilizing intravascular US imaging, it is hard to define a maximum degree of acceptable image distortion due to the periodic scan rotation error, with a periodicity related to the driving frequency. A qualitative judgement of Fig. 7 may show justification of the acceptance of a maximum peak-topeak periodic error angle of $20^{\circ}$.

\section{Working methods of catheter tip scan rotation}

A variety of alternative micro-mechanisms to achieve US element or mirror rotation in the catheter tip has been investigated and generally evaluated on their feasibility (Korbijn 1989). Only a few methods will be discussed here.

\subsection{Flexible drive-shaft rotation transmission}

The direct transmission of a rotational motion from a driving motor through the catheter to the catheter tip is possible using a flexible drive-shaft. This way, an energy or motion transformation in the tip of the catheter can be avoided, but the flexshaft has to meet some seemingly contradicting specifications.

The shaft runs through the whole length of the catheter and adds to its overall bending rigidity. Flexibility of the catheter is mandatory in order to follow the tortuous blood vessels, hence, the driveshaft should also be flexible. For coronary use, the bending rigidity of the catheter is in the order of $30 \mathrm{Nmm}^{2}$. The small diameter of the catheter limits the diameter of the drive-shaft to less than $1 \mathrm{~mm}$. The need for a rotation transmission with a high degree of angular fidelity calls for low friction, rotation symmetrical properties of the driveshaft, low bending rigidity and high torsional rigidity.
For drive-shafts with elastic bending and torsional behaviour it can be shown that reduction of the ratio bending rigidity/torsional rigidity $\left(E I / S_{1}\right)$ improves the angular fidelity (ten Hoff 1993). Two flexible drive-shaft designs will be discussed in the following.

Solid wire or capillary. The simplest flexible drive-shaft is formed by a solid round wire of isotropic material or a capillary, if conducting leads have to run through the shaft. From (Timoshenko and Gere 1973) can be deduced that for such a shaft the ratio $E I / S_{\mathrm{t}}$ is:

$$
\frac{E I}{S_{\mathrm{t}}}=\frac{E I}{G I_{\mathrm{p}}}=\frac{E}{2 G}=1+\nu
$$

where

$E\left(\mathrm{~N} / \mathrm{mm}^{2}\right)=$ Young's modulus,

$I\left(\mathrm{~mm}^{4}\right)=$ moment of inertia of drive-shaft crosssection,

$G\left(\mathrm{~N} / \mathrm{mm}^{2}\right)=$ shear modulus,

$I_{\mathrm{p}}\left(\mathrm{mm}^{4}\right)=$ polar moment of inertia of the round drive-shaft cross-section,

$\nu=$ Poisson's ratio $=E / 2 G-1$,

$S_{\mathrm{t}}=$ torsional stiffness.

Since the value of $\nu$ is restricted to $0.25-0.5$ for all possible isotropic materials, the ratio $E I / S_{\mathrm{t}}$ has a value between 1.25 and 1.5 . For stainless steel, where $\nu=0.32, E I / S_{\mathrm{t}}=1.32$.

It can be shown that this value of $E I / S_{\mathrm{t}}$ is very high. Therefore, this shaft is sensitive to all influences determining the rotation error angle of a shaft in a curved catheter tube. Even under moderately curved conditions solid material driveshafts will not perform well; the catheter has to be kept as straight as possible.

For coronary applications, where the overall catheter bending rigidity should not exceed 30 $\mathrm{Nmm}^{2}$, the diameter of the drive-shaft, being a solid stainless steel wire, should be smaller than 0.2 $\mathrm{mm}$, also allowing for a catheter tube of the same stiffness. The minimum radius of curvature of 15 $\mathrm{mm}$, occurring in the coronary application, re- 
a.

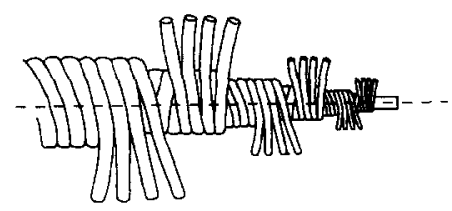

b.
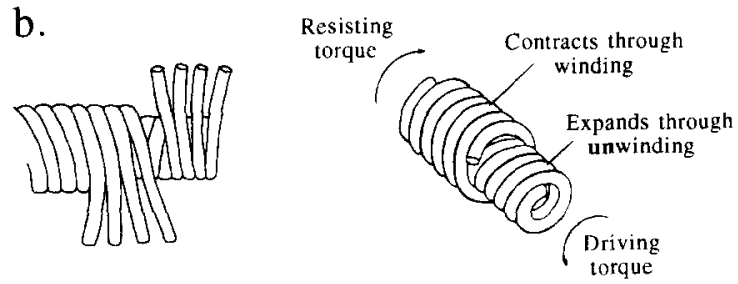

Fig. 10. Spiral type drive-shaft with counter-wound layers of multi-filament spirals. (a) Multi-layer configuration, (b) twolayer configuration (Martin and Johnson 1989).

quires a shaft with a diameter of even less than 0.1 $\mathrm{mm}$, to prevent plastic deformation of the shaft. According to eq. 6, the ratio $E I / S_{1}$ does not depend on the wire diameter, so this value remains at 1.32 . It has been shown that high fidelity rotation transmission cannot be expected using this wire under severely curved conditions.

Spiral-type drive-shaft. A popular design of the flexible drive-shaft is based on the concept of several layers of alternately helically wound steel wire. This concept is known from the literature (Niemann 1981), though mostly for applications with larger diameters $(>3 \mathrm{~mm})$. These spiral-type flexible drive-shafts consist of several layers of multi-filament spirals, where two succeeding layers are wound in opposite directions (Fig. 10a). Essential to good performance of the shaft is the absence of radial space between the spiral layers. The good rotation-transmission characteristics of this shaft, expressed by a low $E I / S_{\mathrm{t}}$ ratio, can be explained by the fact that a torque applied to the shaft in one direction will tend to increase the diameter of one set of spiral layers, while the layers in between, which are counter-wound, tend to decrease in diameter. Both effects partly eliminate each other. The torque is directed largely into pure tensile and compressive stresses in the spiral filaments. This results in a small torque angle of the whole driveshaft assembly, so that the torsional rigidity is much higher than the sum of the torsional rigidities of all individual spirals. In the bending mode the spirals act more independently, so that the bending rigidity is about equal to the sum of the bending rigidities of all individual spirals. The best result is obtained when the driving direction of the shaft induces diameter reduction in the outer layer of spiral filaments.

The small space available in the catheter application limits the construction of the drive-shaft to only two spiral layers (Fig. 10b) (Martin and Johnson 1989). In case of enough interference, i.e. radial pre-tension between the two layers and low driving torque, the driving direction will not interfere with the drive-shaft characteristics and rotation transmission fidelity may be expected to be the same for both driving directions. The $E I / S_{\mathrm{t}}$ ratio for a spiral-type drive-shaft of small dimensions (OD $<1 \mathrm{~mm}$ ) has been shown to be less than 0.015 .

A constant error angle in the order of $10^{\circ}$ has been shown to be possible in normal clinical application. Also, a periodic error angle $<20^{\circ}$ peak-topeak is feasible. A stochastic error angle $<1^{\circ} \mathrm{im}$ plies that the stochastic variation of the torque is less than $10 \%$ of the constant torque and also less than $10 \%$ of the amplitude of the periodic variation of the torque. These demands seem reasonable and can be met. Consequently, the set requirements can be met by this flexible driveshaft.

\subsection{Electrostatic micro-motor}

In conventional technology the electrostatic motor hardly finds any application, because the maximum torque of the motor is more than four orders of magnitude lower than the torque of an electro-magnetic motor of the same dimensions (Bart et al. 1988), due to the limited breakdown voltage in such a motor. However, for very small motors with very small air gaps down to $2 \mu \mathrm{m}$, the maximum electric field can be 50-100 times higher before breakdown occurs.

Therefore, the theoretical torque generated between rotor and stator may well be large enough to drive an acoustic mirror in water. But many basic and practical problems are to be solved before practical utilization of the electrostatic concept. 


\subsection{Electro-magnetic micro-motor}

A micro-motor based on conventional precision engineering technology is in closer reach (Hadfield 1992). Prototypes of a motor with a diameter of 1 $\mathrm{mm}$ have been produced successfully and have been shown to be capable of driving a small acoustic mirror in water. A rotor of permanent magnetic material, with a diameter of $0.7 \mathrm{~mm}$, rotates in two bearings, driven by a stator with currentconducting leads. In a first prototype the stator conductors consisted of four seven-fold coils of thin copper wire. The production of these coils, the positioning in the stator and the connection to the motor-leads turned out to be critical. Also, the resulting distribution of conductors around the rotor was sub-optimal. Therefore, a flex-print based stator with a more optimal configuration of conductor leads has been developed. This concept is easier to reproduce and provides better perspectives for economical manufacturing and further miniaturization (Lancée and Bom 1993).

A periodic error angle of less than $10^{\circ}$ peak-topeak can be achieved at a rotational speed of 3600 $\mathrm{rev} . \mathrm{min}$. Catheter tip prototyping, using this motor to drive an acoustic mirror, is now in process.

\section{Conclusions}

In intravascular ultrasound imaging, radial and tangential catheter tip motion with respect to the vessel wall give rise to image artifacts and, in particular for in vitro experiments, immobilization of the catheter tip should be ensured.

Deviation from a predictable uniform scan rotation also causes image artifacts, exhibiting either loss of absolute orientation (due to a constant error angle), loss of quality of the real-time aspect (due to a stochastic error angle), or the occurrence of deceptive image distortions (due to a periodic error angle). The magnitude of acceptable scan rotation errors (actual scan rotation angle minus expected angle) depends on the specific clinical application. But in general, the stochastic error should be kept to a minimum: random misplacement of image lines in the order of degrees is already disturbing to the interpretation of the image. As an arbitrary criterion for the periodic error angle, a maximum peak-to-peak value of $20^{\circ}$ can be defined.

The feasibility of catheter tip driving mechanisms for rotation of the ultrasonic beam should be investigated, considering their potential effects on the image reliability. A $0.8 \mathrm{~mm}$ OD doublelayer spiral type flexible drive-shaft has been developed, which meets the requirements, exhibiting a bending rigidity of $1.5 \mathrm{Nmm}^{2}$ and a torsional rigidity of more than $100 \mathrm{Nmm}^{2}$. This drive-shaft is 90 times less sensitive to influences, inducing rotation error angles, as a simple wire of stainless steel, for example.

For clinical applications, spiral drive-shaft catheters are being used in many research institutes. It is apparent that the absence of the long drive-shaft, retaining good image quality for all catheter geometries, is of importance to catheter handling and flexibility of catheter design. Therefore, the 'motor in the tip' approach is potentially promising.

For future application, an electro-magnetic micromotor has been developed, with a diameter of $1 \mathrm{~mm}$, for driving an acoustic mirror in the catheter tip. The motor exhibits adequate running behaviour under experimental conditions. First in vitro images have been documented.

\section{Acknowledgements}

This research has been financially supported by the Netherlands Technology Foundation and the Dutch Ministry of Economic Affairs. Practical support by and fruitful co-operation with the division of Precision Engineering of the Technical University of Delft and Du-MED B.V., Rotterdam, both in The Netherlands and with USCI, C.R. Bard Inc., Billerica MA, USA, are gratefully acknowledged.

\section{References}

Bart SF, Lober ThA, Howe RT, Lang JH, Schlecht MF. Design considerations for micromachined electric actuators. Sensors Actuators 1988; 14: 269-292.

Bom N, ten Hoff H, Lancée CT, Gussenhoven WJ, Bosch JG. Early and recent intra-luminal ultrasound devices. Int $\mathbf{J}$ Card Imaging 1989; 4: 79-88.

Bom N, Roelandt JRTC. Intravascular ultrasound: newest 
branch on the echo-tree. Cardiovasc Imaging 1992; 4: 55-59.

Bom N, Lancée CT, Gussenhoven EJ, Li W, ten Hoff H. Basic principles of intravascular ultrasound imaging. In: Tobis JM, Yock PG, eds. Intravascular ultrasound imaging. New York: Churchill Livingstone, 1992: 7-15.

Finet G, Maurincomme E, Tabib A, Crowley RJ, Magnin I, Roriz R, Beaune J, Amiel M. Artifacts in intravascular ultrasound imaging: Analysis and implications. Ultrasound Med Biol 1993; 19: 533-547.

Hadfield P. Tiny motor clears way for microsurgery. New Scientist 1992; 136 (Oct. 10): 19.

ten Hoff H, Korbijn A, Smit ThH, Klinkhamer JFF, Bom N. Imaging artifacts in mechanically driven ultrasound catheters. Int J Card Imaging 1989; 4: 195-199.

ten Hoff H. Scanning mechanisms for intravascular ultrasound imaging: a flexible approach. PhD thesis. Erasmus University Rotterdam 1993: 115. (ISBN 90-9006072-3)

Korbijn A. Onderzoek naar alternatieve aandrijvingen voor een echo-imaging catheter. MSc thesis, Technical University Delft, Department of Mechanical Engineering, Precision Engineering Section, 1989; Report A-489/K14. de Kroon MGM, Van der Wal LF, Gussenhoven EJ, Bom N. Angle-dependent backscatter from the arterial wall. Ultrasound Med Biol 1991; 17: 121-126.

Lancée CT, Bom N. Ultrasonic instrument with a micro-motor having stator coils on a flexible circuit board. US patent No. 5.240.003, Aug. 31, 1993.

Li W, Gussenhoven WJ, Zhong Y, The SHK, Di Mario C, Madretsma S, Van Egmond FC, de Feyter P, Van Urk $H$, Rijsterborgh $\mathbf{H}$, Bom N. Validation of quantitative analysis of intravascular ultrasound images. Int $\mathrm{J}$ Card Imaging 1991; 6: 247-253.

Martin RW, Johnson CC. Design characteristics for intravascular ultrasound catheters. Int J Card Imaging 1989; 4: 201-216.

Niemann G. Maschinen-elemente, Band I. Berlin: SpringerVerlag, 1981: 356.

Timoshenko SP, Gere JM. Mechanics of materials. Converted version into SI units. New York: Van Nostrand Reinhold Company, 1973: pp 61, 87, 135. 